1989-09-01

\title{
Interactive Measurement of Three-Dimensional Cardiac Morphology
}

William A. Barrett

william_barrett@byu.edu

Shawn C. Becker

Follow this and additional works at: https://scholarsarchive.byu.edu/facpub

Part of the Computer Sciences Commons

\section{Original Publication Citation}

Shawn C. Becker and William A. Barrett: "Interactive Measurement of Three-Dimensional Cardiac Morphology." IEEE Proceedings of Computers in Cardiology, pp. 429-432, Jerusalem, September, 1989.

\section{BYU ScholarsArchive Citation}

Barrett, William A. and Becker, Shawn C., "Interactive Measurement of Three-Dimensional Cardiac Morphology" (1989). Faculty Publications. 737.

https://scholarsarchive.byu.edu/facpub/737 accepted for inclusion in Faculty Publications by an authorized administrator of BYU ScholarsArchive. For more information, please contact ellen_amatangelo@byu.edu. 


\title{
Interactive Measurement of Three-Dimensional Cardiac Morphology
}

\author{
Shawn C. Becker and William A. Barrett \\ Department of Computer Science \\ Brigham Young University
}

\section{Summary}

Techniques have been developed for extracting three-dimensional (3D) measurements directly and interactively from 3D representations of Cine CT images. In order to obtain $3 \mathrm{D}$ measurements the anatomical surface is interrogated directly through the use of a 3D graphical "probe" (line). The probe is interactively positioned and stretched between any visible surface points to allow direct capture of $3 D$ (linear) distance. Distance measurements are updated in real time as the probe dynamically "penetrates" any intervening anatomy while providing valuable depth cuing for visual feedback and probe placement. Curvilinear distance is obtained by stretching the probe over multiple surface points. The probe is implemented using a general z-buffer and is therefore applicable to a variety of $3 \mathrm{D}$ imaging techniques.

\section{Introduction}

Recent advancements in medical imaging technology (eg. CT, Nuclear Medicine, Ultrasound, Cine $C T$, and MRI) have led to the development of a variety of algorithms for three-dimensional (3D) visualization of anatomical surfaces or structures contained within the imaged volume. Some of these algorithms are based on an explicit description of the surface anatomy while others make use of recently popularized volume rendering techniques. Previous work ${ }^{1,2}$ describes techniques for automated extraction and interactive visualization of 3D (and 4D) cardiac surface anatomy from any arbitrary viewpoint. The resulting surface images greatly enhance understanding of changes in 3D shape, size, and spatial interrelationships (ie. morphology) of cardiac structures in both normal and diseased states.

While visualization of $3 \mathrm{D}$ and $4 \mathrm{D}$ cardiac surface anatomy is an important adjunct to diagnosis, interactive measurement of static or dynamic surface geometry is equally important. Accordingly, the purpose of this paper is to describe some techniques for interactive measurement of $3 \mathrm{D}$ cardiac morphology.
Methodology

In order to obtain morphological measurements the anatomical surface image is interrogated directly through the use of a 3D graphical "probe" (line). The probe is implemented using a 3D DD face-connected line-generating algorithm and a $\mathrm{z}$ (depth) buffer which represents the surface position from any given viewpoint. Comparison of the DDA line with the $\mathrm{z}$ buffer allows the probe to be interactively positioned and stretched between any two visible surface points for direct measurement of 3D (linear) distance. The probe also provides real-time visual feedback pertaining to object geometry and surface dimensions.

\section{Probe Implementation}

A visible point on the object's surface is specified with a 2D mouse-driven cursor and identifies one end of the probe. A second point which is dragged along the surface identifies the other end of the probe. Hence, the probe functions like a $3 \mathrm{D}$ rubber band line which can be stretched about an object's surface using a mouse-driven cursor that moves on the $2 \mathrm{D}$ view plane while the probe visually penetrates intervening surface structures as it is updated in real-time.

Proper visual feedback demands that portions of the probe be hidden when intersecting intervening surface structures.

Visualization of this sort can be implemented easily when a depth buffer is used to define the surface. A depth buffer, is the by-product of a variety of surface rendering algorithms ${ }^{3}$. When a scene is orthographically projected, the pixel coordinates and associated depth value are simply the result of the surface point's world coordinates being sent through the view transformation $T$. Mouse-defined probe end points $(A$ and $B$ ) in view plane coordinates (Figure 1) have corresponding depth values $\left(A_{Z^{\prime}} B_{Z}\right)$ obtainable directly from the depth buffer. A modified Digital Differential Analyzer algorithm 4 is used to interpolate $\left(\mathrm{x}_{\mathrm{v}}, \mathrm{y}_{\mathrm{v}}\right)$ pixels and associated $\left(\mathrm{z}_{\mathrm{v}}\right)$ depth values between the end points. 


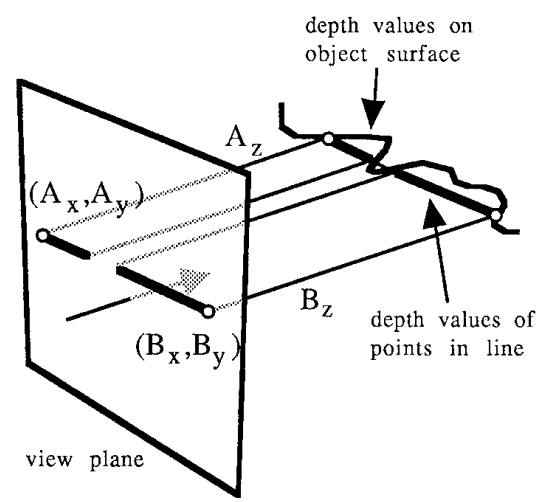

Figure 1. Probe visualization using depth buffer described surface.

To make the probe appear to penetrate the surface the principal for normal depth buffering is used when drawing the line in 2D image space: the line is visible only when line depth values are less than the surface depth. The algorithm for probe visualization and movement on a precomputed depth buffer is listed below in Figure 2 .

\section{Probe Measurements}

When view space coordinate information $\left(\mathrm{x}_{\mathrm{v}}, \mathrm{y}_{\mathrm{v}}, \mathrm{z}_{\mathrm{v}}\right)$ provided by the depth buffer is applied to the inverse view transformation $\left(\mathrm{T}^{-1}\right)$, original surface point coordinates in world space $\left(\mathrm{x}_{\mathrm{w}}, \mathrm{y}_{\mathrm{w}}, \mathrm{z}_{\mathrm{w}}\right)$ can be recalculated and used to obtain the distance between two object surface points $\left(|| P_{w 1}-P_{w 2}||\right)$.

However, since linear distance is a relative measure between two points and orthographic transformations result in a scale-factored relative distances, view space coordinates derived from orthographic projections may be used directly for determining the distance between two visible surface points. Total reverse transformation is unnecessary when calculating distances from an orthographically projected depth buffer. To convert the relative pixel measurement obtained from the depth buffer to useful world units the pixel based distance is scaled by the scale factor used in device normalization (S).

$$
|| P_{w 1}-P_{w 2}||=S|| P_{v 1}-P_{v 2}||
$$

$$
\text { where } \mathbf{S}=\frac{\text { world window dimensions }}{\text { view window dimensions in pixels }}
$$

Linear measurements using this approach have been shown to be both accurate (limited by scene and image resolution) and reproducible when taken from different orientations.

\section{Probe visualization during reorientation}

One consequence of using a depth buffer to interact with the surface is that only visible portions can be used to define probe end points. However, if a desired point on the surface is not visible in one view, the view may be interactively changed (using a precomputed set of views ${ }^{2}$ until the point is visible. Surface probes can easily be updated during real-time view reorientation to appear statically attached to the object's surface. In this case, the full reverse view transformation is used to obtain world coordinates of probe end points which are then forward transformed into the new view for rerendering.

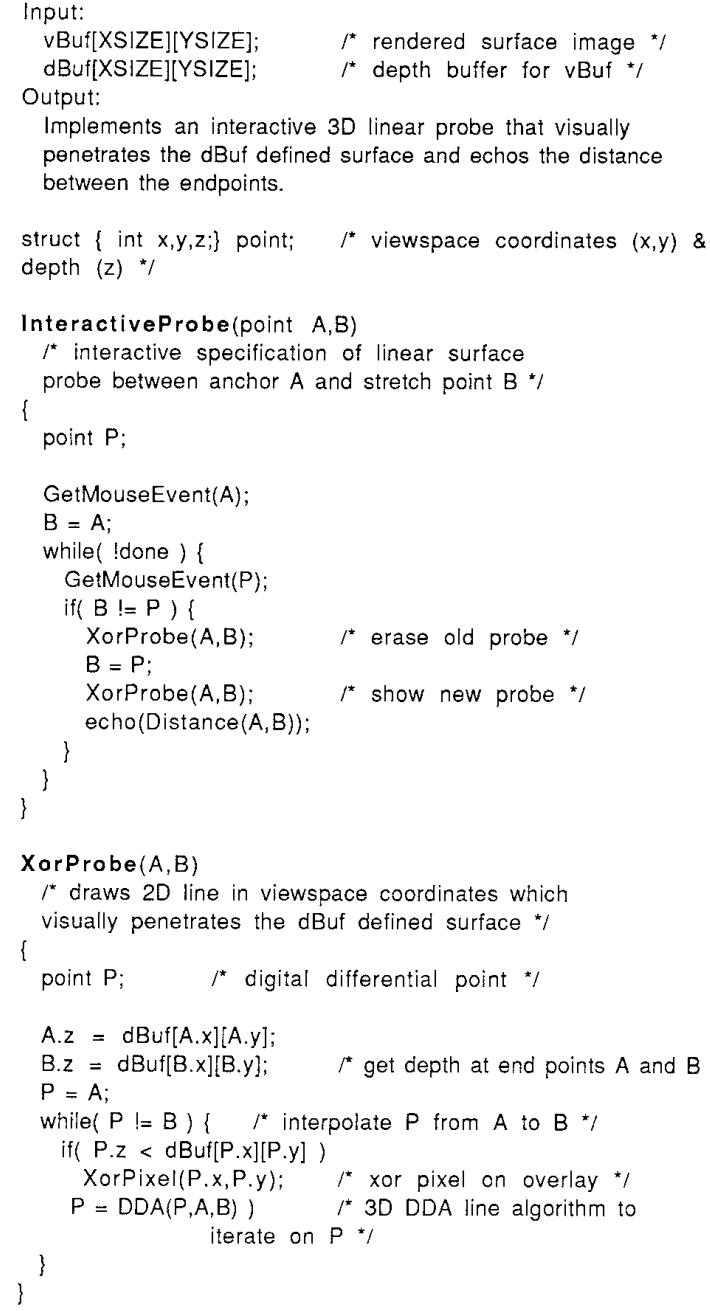

Figure 2. C-code for interactive probe visualization and measurement of 3D distance. 


\section{Curvilinear measurements}

The next logical variation of probe-based object measurements is to support interactive curve measurements. One approach involves summing the linear distances between multiple user-defined surface points. A series of end-connected linear probes can be used to calculate piecewise linear curve distance. Another curve measurement technique amounts to projecting a probe onto the surface of the object. Each pixel in the probe has corresponding

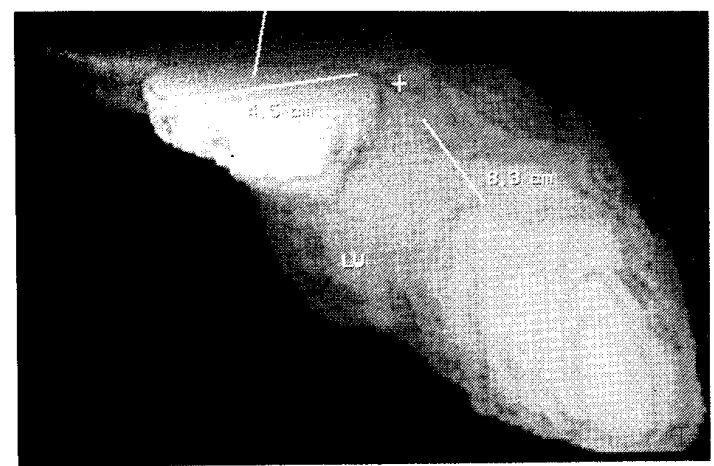

Figure 3a. Probe measurements at end-diastole.

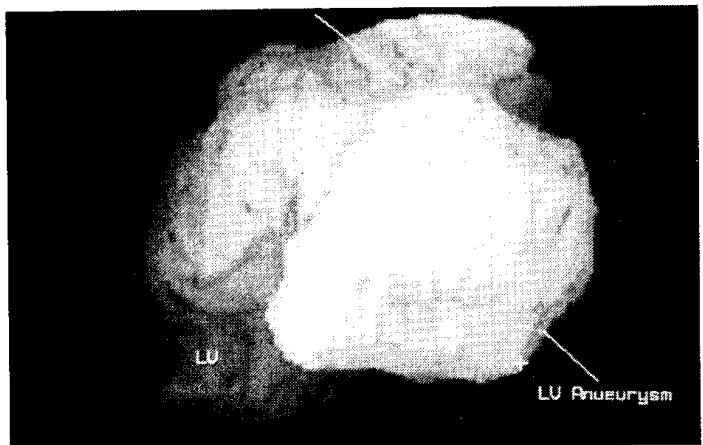

Figure 3c. Measurement of aneurysm dimensions.

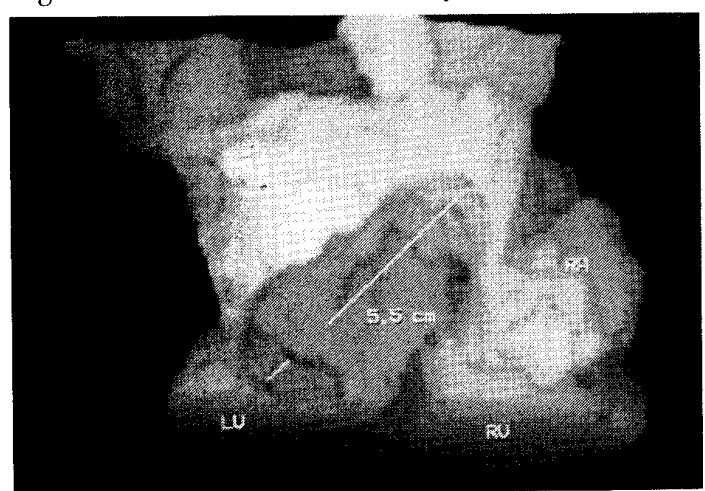

Figure 3e. Tumor cavity - length. surface point coordinate information available in the depth buffer. Accurate curve distance can be thus calculated by summing linear distance between pairs of visible surface points in the probe's path.

$$
\text { Length }_{\text {curvilinear }}=S \sum_{i=1}^{n}|| P_{i}-P_{i-1}||
$$

The piecewise linear method may be appropriate for taking point to point cumulative approximations of

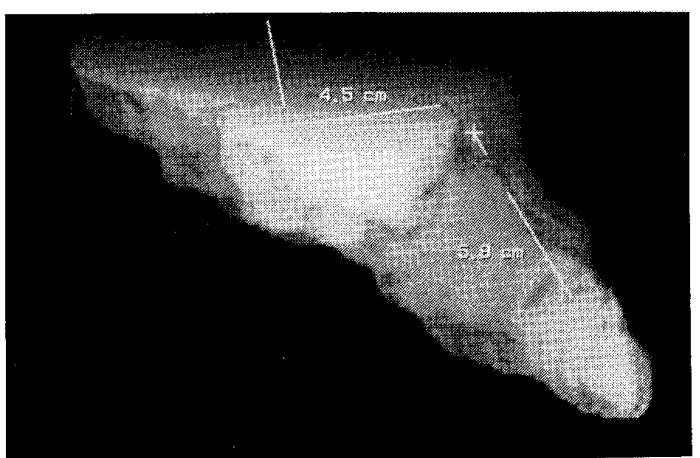

Figure $3 b$. Probe measurements at end-systole.

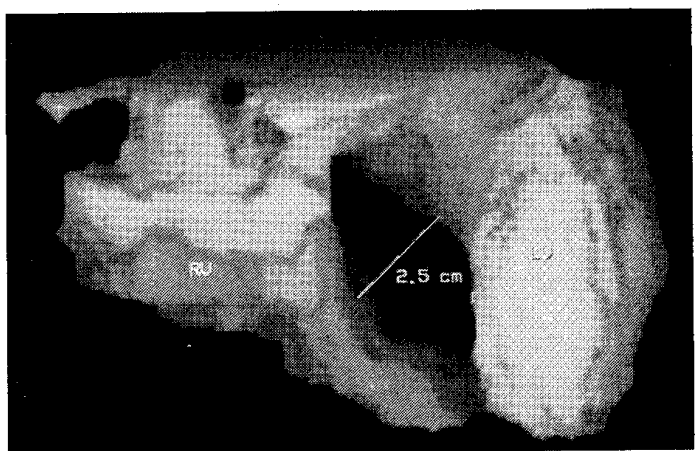

Figure 3d. IHSS - septal-muscle region.

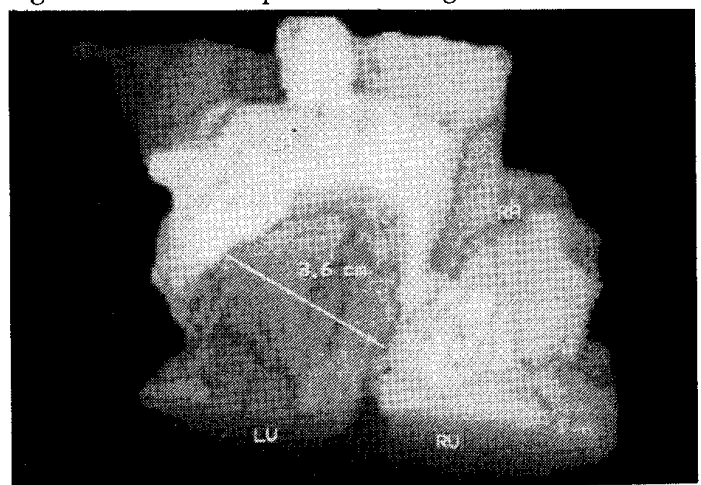

Figure 3f. Tumor cavity - width. 
object geometry dimensions. The projected curve method, which may be likened to laying a measuring tape directly on the object's surface, provides more precise circumferential measurements.

\section{Area measures}

Area measurements may be obtained by calculating the area between a given probe and its projected surface curve. Using the depth buffer this can be done by summing surface to probe depth differences over all $n$ points in the probe when the probe line is visible and then adjusting for discretization in the view plane.

$$
\begin{aligned}
& \text { Area }_{\text {probe-curve }}=\mathrm{S}^{2} \mathrm{~N} \sum_{\mathrm{i}=1}^{\mathrm{n}} \max \left(\left(\mathrm{z}_{\mathrm{pi}}-\mathrm{z}_{\mathrm{ci}}\right), 0\right) \\
& \mathrm{N}=\frac{\text { true probe length }}{\text { discrete probe length }}=\frac{|| \mathrm{P}_{\mathrm{n}}-\mathrm{P}_{1}||}{\mathrm{n}} \\
& \underline{\text { Results }}
\end{aligned}
$$

3D measurements were extracted interactively from a variety of cardiac pathologies shown in Figure 3 . Figures $3 a$ and $3 b$ show a normal left ventricle in the RAO $18^{\circ}$ projection at end-diastole (a) and endsystole (b). End points for the probe extending from the anterior base to the apex of the ventricle are indicated with a cross and define a 3D linear distance of $8.3 \mathrm{~cm}$. Note that portions of the probe are "buried" (ie. invisible) due to the curvature of the ventricle. The same base-to-apex measurement was made at end-systole (Figure $3 \mathrm{~b}$ ) and indicates a percent shortening of $5.8 / 8.3=.7$. Probe measurements such as these can be extracted interactively and in real time.

Figure $3 c$ demonstrates one of a variety of dimensional measurements obtained from a large posterior ventricular aneurysm. Figure $3 \mathrm{~d}$ shows an IHSS study from the anterior position at end-diastole. Extraction of the 3D distance $(2.5 \mathrm{~cm})$ between the right and left ventricles allows the dimensions of the hypertrophied muscle in the septal region to be quantified.

The posterior view of the blood-contrast surface in Figures $3 e$ and $3 f$ shows a cavity caused by a left atrial tumor. Using the 3D measurement technique the dimensions of the cavity can be quantified interactively in any arbitrary direction.

The ability to measure anatomical morphology (ie. shape, size, and spatial interrelationships) directly and interactively from the surface image provides a more natural, efficient, and precise diagnostic tool than conventional measurement techniques which are limited to point placement within or between planar or axial images, while the latter provide a basis for validation of the new methodology. Measurement of
3D distance allows quantification of chamber dimensions over time from any orientation and provides improved discrimination between normal and abnormal ventricular shapes, sizes, and wall motion parameters. As a result, visualization of $3 \mathrm{D}$ surface anatomy is greatly enhanced through the addition of graphical probing tools for interactive measurement.

\section{Conclusions}

A technique has been presented for direct interactive measurement of 3D cardiac morphology using a graphical probe. This simple morphometric tool enhances visualization by supporting direct interactive measurement of $3 \mathrm{D}$ cardiac surface anatomy. In addition to measurement, the visual feedback provided by this simple technique communicates much about the structure of the surface. Furthermore, implementation of the probe using a general depth buffer makes it applicable to a variety of surface or volume rendering techniques.

Depth buffers are commonly used to solve hidden surface problems in the rendering of objects contained within image volumes. However the depth buffer provides additional utility during interactive visualization and measurement when it is used to define the visible surface of the objects. Without ever accessing or changing the data within the image volume, probes and curves may be interactively specified and positioned on the object's surface. These tools provide valuable visual cues and distance measures that extend the observer's ability beyond mere "seeing" of 3D structure to virtually "touching and feeling" or quantifying cardiac morphology.

\section{$\underline{\text { References }}$}

1. Barrett, W.A. and Udupa, J.K: A Low-Cost PCBased Image Workstation for Dynamic Interactive Display of Three-Dimensional Anatomy", SPIE Medical Imaging, Newport Beach, (1989).

2. Barrett, W.A. and Udupa, J.K.: Dynamic Display and Quantitative Analysis of Three-Dimensional Left Ventricular Pathology, Proceeding of the IEEE, Computers in Cardiology, Bethesda, MD, 1988.

3. Chen, L., Herman, G.T., Reynolds, A, and Udupa, J.K.: Surface Shading in the Cuberille Environment, IEEE Computer Graphics and Applications, December, 33-43 (1984).

4. Field, D.: Incremental Linear Interpolation", ACM Transactions on Graphics, (1):1-11 (1985). 